\title{
Soluble receptor for advanced glycation end products in critically ill patients and its associations with other clinical markers and 28-day mortality
}

\author{
This article was published in the following Dove Press journal: \\ Clinical Interventions in Aging \\ 19 November 2014 \\ Number of times this article has been viewed
}

\author{
Yanzi Cheng' \\ Jiwen Zhong' \\ Yang Xiang' \\ Fan Zeng' \\ Dehong $\mathrm{Cai}^{2, \dagger}$ \\ Ling Zhao' \\ 'Department of Intensive \\ Care Unit, Zhuhai People's \\ Hospital, Zhuhai, Guangdong, \\ People's Republic of China; \\ ${ }^{2}$ Department of Endocrinology, \\ Zhujiang Hospital of Southern Medical \\ University, Guangzhou, Guangdong, \\ People's Republic of China \\ ${ }^{\dagger}$ Dehong Cai passed away on \\ April 19, 2014
}

\begin{abstract}
Purpose: To investigate the possible associations between serum levels of soluble receptor for advanced glycation end products (sRAGE) and specific clinical markers and prognosis in critically ill patients diagnosed with stress hyperglycemia.

Patients and methods: A total of 70 critically ill patients and 25 normal controls were recruited for this study. Serum levels of sRAGE and advanced glycation end products (AGEs) were determined using enzyme-linked immunosorbent assay. Additional data on other clinical markers were obtained from patient records in the intensive care unit. Comparisons of sRAGE and AGEs levels between groups were assessed by $t$-test. The relationships between sRAGE and other clinical markers were assessed by Pearson's correlation analyses and multiple linear regression analyses. Risk factors for prognosis, such as 28-day mortality were analyzed using logistic regression analysis.
\end{abstract}

Results: Serum sRAGE and AGEs levels were significantly higher in critically ill patients, compared to normal controls $(P<0.05)$. The increase in serum sRAGE levels was significantly correlated with AGEs levels, interleukin-6 levels, and the sequential organ failure assessment score $(P<0.01)$. Using multiple linear regression analysis, the association between AGEs and sRAGE remained significant after adjustment of other clinical factors. However, there were no significant correlations between sRAGE levels and patient outcome in these critically ill patients.

Conclusion: Serum sRAGE levels were significantly elevated in critically ill patients and positively correlated with higher AGEs levels, but sRAGE levels were not associated with increased mortality, suggesting sRAGE levels are not a predictor of prognosis in critically ill patients.

Keywords: soluble receptor for advanced glycation end products, advanced glycation end products, critically ill patients, predictor, relationship

\section{Introduction}

Advanced glycation end products (AGEs) are stable covalent, structurally diverse complexes that are formed when macromolecules, including proteins, lipids, and nucleic acids, undergo spontaneously non-enzymatic glycation with glucose and other reducing monosaccharides. ${ }^{1,2}$ AGEs formation is chemically irreversible, so that once formed, these compounds will accumulate in the tissues continuously. AGEs have been linked to a wide array of complications and diseases, and mediate their effects by binding to the specific cell-surface receptor for advanced glycation end products (RAGE). Upon interaction with RAGE, multiple signaling pathways are activated, which induce oxidative stress and inflammation, cytokine release, stimulation of 
the coagulation cascade, and increase in lipid metabolism, leading to a series of pathophysiological changes. ${ }^{3-5}$

RAGE is a member of the immunoglobin superfamily of cell surface receptors and is involved in immune response signaling. Soluble RAGE (sRAGE), a newly identified subtype of RAGE, is considered a truncated isoform of RAGE. Specifically, sRAGE is formed after undergoing proteolytic cleavage to remove the intracellular domain and is therefore incapable of inducing intracellular signal transduction. However, after binding with AGEs, sRAGE can inhibit interactions of AGEs with the full-length RAGE, thereby further reducing the RAGE-induced signal transduction pathway. Thus, sRAGE serves in a protective manner, antagonizing endogenous RAGE-mediated intracellular damage, and may be used as a biomarker to reflect pathological status in the body. $1,3,4,6$

Recent studies have shown that sRAGE levels are elevated in patients with type 1 diabetes, type 2 diabetes, and diabetic nephropathy, but decreased in patients with coronary artery disease, atherosclerosis, hypertension, chronic obstructive pulmonary disease, heart failure, and hyperlipidemia..$^{7-11}$ Moreover, increased AGEs levels are reflected in higher expression of RAGE and sRAGE. ${ }^{7-10}$ As a result of continuous stimulation by chronic hyperglycemia, AGE levels are significantly higher in diabetic patients, compared to normal controls. Chronic formation and accumulation of AGEs has been demonstrated to be an important factor in diabetic complications, ${ }^{12}$ while increased sRAGE levels have a protective effect for diabetic conditions, including macrovascular complications. ${ }^{8}$ Previous studies investigating sRAGE mainly focused on patients with chronic diabetes. However, it is still unclear whether there are any changes in AGE and sRAGE levels under the state of acute stress hyperglycemia.

Stress hyperglycemia is common in critically ill patients, and is associated with increased rates of morbidity and mortality among the patient population. Life-threatening situations cause an intense stress response combined with stress hyperglycemia, which induce severe oxidative stress injuries and inflammatory reactions, promoting serious substance and energy metabolic disorders. Thus, stress hyperglycemia is an important marker of metabolic disorders in critically ill patients. Increased severity of illness is associated with an increased stress reaction, resulting in higher incidence of stress hyperglycemia. Previous studies have reported the incidence of stress hyperglycemia ranging from 50\% to $80 \%$ in intensive care units (ICUs).$^{13}$ Because hyperglycemia in the presence of oxidative stress and inflammation promotes glycoxidation of intracellular and extracellular proteins,
AGEs continue to form and accumulate in the tissues. ${ }^{14}$ We hypothesize that oxidative stress and inflammation in critically ill patients result in an increased production of AGEs, which may in turn lead to elevated expression of sRAGE. Therefore, this study was designed to measure serum AGEs and sRAGE levels in ICU patients, and make a comparison with levels in normal controls. In addition, we investigated the associations of sRAGE levels with specific clinical markers and mortality from the ICU to explore their potential correlations and clinical relevance.

\section{Material and methods}

\section{Study population}

This observational study was carried out in a universityaffiliated teaching hospital (Zhuhai hospital, Jinan university, Zhuhai, Guangdong, the People's Republic of China) with an ICU with a mixed medical-surgical closed-format setting with 15 beds. A total of 70 critically ill patients with hyperglycemia (43 men and 27 women; mean age 57.01 \pm 17.14 years admitted to the comprehensive ICU between January 2012 and July 2013 were included in this study. Study inclusion was based upon a minimum ICU stay of 3 days, receipt of an acute physiology and chronic health evaluation (APACHE-II) score higher than 15, age over 18 years, and no medical history of diabetes. Patients with brain death, end-stage chronic diseases, diabetes, or terminal cancer were excluded. A total of 25 healthy subjects ( 15 men and 10 women; mean age 55.41 ( \pm 10.42 years) who received a regular physical evaluation during the same period were enrolled as the control group. The controls were matched to the cases by sex and age as frequently as possible. The inclusion criteria for the normal controls were age 18 years or older and normal blood glucose levels (fasting plasma glucose $<6.0 \mathrm{mmol} / \mathrm{L}$ and 2-hour glucose in the oral glucose tolerance test $<7.8 \mathrm{mmol} / \mathrm{L}$ ). The normal controls with liver and kidney dysfunction, cardiovascular disease, diabetes, or tumor were excluded. Signed informed consent was obtained from each subject who participated in this study. For patients who were not physically able to sign, their direct relatives or guardians signed the form with permission. The study was approved by the ethics committee of Zhujiang Hospital (Guangzhou, People's Republic of China).

\section{Measurement of serum sRAGE, AGEs, and interleukin- 6 serum levels}

Fasting blood samples without anticoagulant were collected in the morning and within 24 hours after admission. The samples were prepared by centrifuging at 3,000 rev/min 
for 10 minutes, separating the serum, and then storing at $-80^{\circ} \mathrm{C}$ prior to analysis. Serum levels of sRAGE, AGEs, and interleukin (IL)-6 were determined by enzyme-linked immunosorbent assay. The human sRAGE kits used in this study (R\&D Systems, Inc., Minneapolis, MN, USA) had an inter-assay coefficient of variation $<10 \%$ and an intra-assay coefficient of variation $<4 \%$. AGE and IL-6 levels were measured using human AGEs and IL-6 kits (CUSABIO, Wuhan, People's Republic of China), and were performed as previously described with each unit of AGEs corresponding to $2 \mu \mathrm{g}$ of glyceraldehyde-derived AGE-bovine serum albumin as the standard. ${ }^{15}$

\section{Measurement of other clinical markers}

Serum creatinine, C-reactive protein, and other biochemical markers were measured by the Hitachi 7,600 automatic biochemical analyzer (Hitachi Ltd., Tokyo, Japan). Rapid blood glucose levels were detected by a blood glucose meter and blood glucose test strips (Roche, Basel, Switzerland). Glycated hemoglobin $\left(\mathrm{HbA}_{1 \mathrm{c}}\right)$ was measured by highperformance liquid chromatography using an automated glycated hemoglobin analyzer (Tosoh, Yamaguchi, Japan), with the normal reference range of $4.2 \%-6.2 \%$. In addition to these biochemical markers, patients' APACHE-II scores and sequential organ failure assessment (SOFA) scores were measured and recorded within 24 hours of arrival in the ICU as well as 28-day mortality. Patients were then screened for stress hyperglycemia upon arrival as defined by no history of diabetes and fasting blood glucose $>6.9 \mathrm{mmol} / \mathrm{L}$ or random blood glucose $>11.1 \mathrm{mmol} / \mathrm{L}$, and $\mathrm{HbA}_{1 \mathrm{c}}<6.5 \%{ }^{16}$

\section{Statistical analysis}

All statistical analyses were performed using the SPSS 16.0 statistical software (SPSS Inc, Chicago, IL, USA). $P$-values less than 0.05 were considered statistically significant. The results are expressed as mean and standard deviation (mean \pm standard deviation), and independent samples $t$-test were used for between-group comparisons. To investigate the relationships between sRAGE and other clinical markers, Pearson's correlation analyses and multiple linear regression analyses were performed. Risk factors for prognosis such as 28-day mortality were analyzed using logistic regression analysis.

\section{Results}

\section{Clinical data of critically ill patients}

Among the 70 patients included in the study, the average APACHE-II score was $25.30 \pm 5.75$ and the average ICU
Table I Baseline characteristics and outcome of patients admitted in the ICU

\begin{tabular}{ll}
\hline Characteristics & ICU patients \\
\hline $\mathrm{N}$ & 70 \\
Age, years & $57.0 \mathrm{I} \pm \mathrm{I} 7.14$ \\
Sex $(\%$ male) & $43.00(6 \mathrm{I} .42)$ \\
APACHE-II score & $25.30 \pm 5.75$ \\
SOFA score & $12.87 \pm 2.87$ \\
AGEs, U/mL & $13.85 \pm 3.63$ \\
sRAGE, $\mathrm{ng} / \mathrm{mL}$ & $\mathrm{I} .63 \pm 0.53$ \\
IL-6, pg/mL & $75.84 \pm 35.27$ \\
Glucose, $\mathrm{mmol} / \mathrm{L}$ & $12.24 \pm 2.4 \mathrm{I}$ \\
HbA ${ }_{\text {Ic }} \%$ & $5.59 \pm 0.64$ \\
$\mathrm{C}-$ reactive protein, $\mathrm{mg} / \mathrm{L}$ & $91.26 \pm 42.04$ \\
Creatinine, $\mu \mathrm{mol} / \mathrm{L}$ & $\mathrm{I} \mathrm{I} .66 \pm 85.09$ \\
ICU acquired sepsis, \% & $32.00(45.7 \mathrm{I})$ \\
ICU duration, days & $14.74 \pm 6.62$ \\
ICU mortality, \% & $18.00(25.7 \mathrm{I})$ \\
\hline
\end{tabular}

Notes: Values are mean \pm standard deviation for continuous variables and number (\%) for categorical variables.

Abbreviations: AGEs, advanced glycation end products; APACHE, acute physiologic score and chronic health evaluation; $\mathrm{HbA}_{1 \mathrm{lc}}$, glycated hemoglobin; ICU, intensive care unit; IL-6, interleukin-6; SOFA, sequential organ failure assessment; sRAGE, soluble receptor for advanced glycation end products.

duration was $14.74 \pm 6.62$ days. A total of 18 patients died, giving a mortality rate of $25.71 \%$ (Table 1 ).

\section{Comparison of serum AGEs and sRAGE levels between critically ill patients and normal controls}

As shown in Figures 1 and 2, serum AGEs and sRAGE levels in critically ill patients were significantly higher than those found in

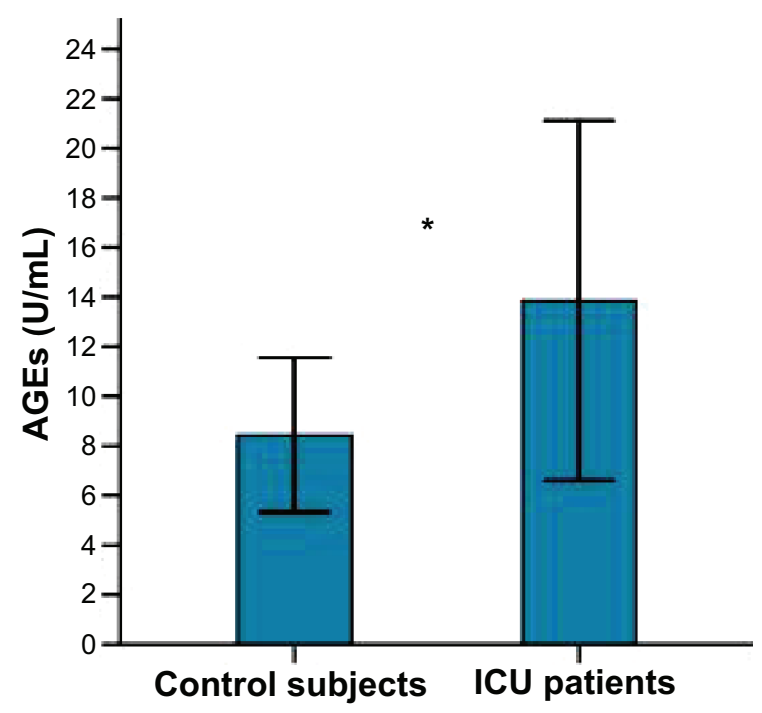

Figure I AGEs levels in normal controls and critically ill patients. Note: $* P<0.001$.

Abbreviations: AGEs, advanced glycation end products; ICU, intensive care unit. 


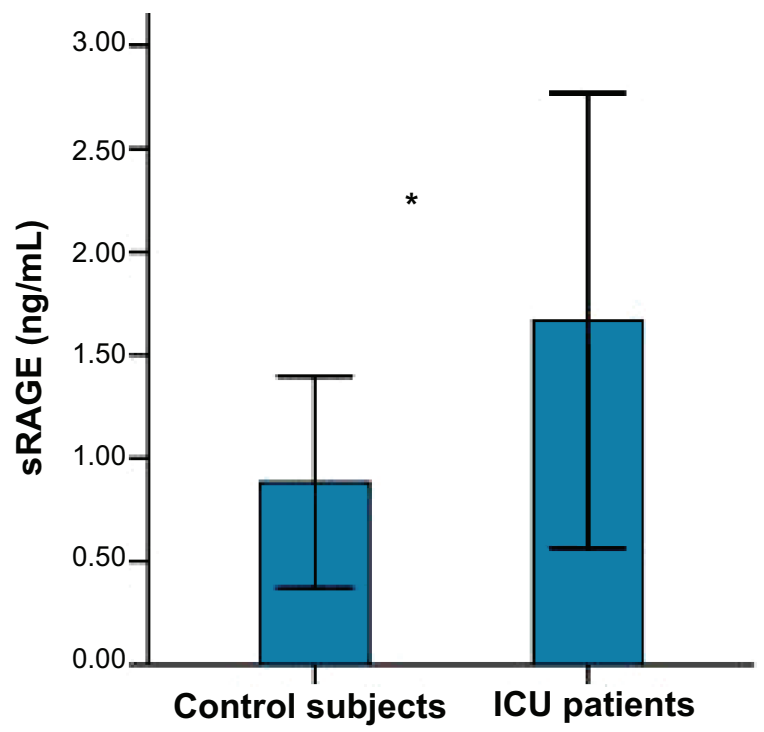

Figure 2 sRAGE levels in critically ill patients and normal controls. Note: $* P<0.001$.

Abbreviations: sRAGE, soluble receptor for advanced glycation end products; ICU, intensive care unit.

the normal controls $(13.85 \pm 3.63 \mathrm{U} / \mathrm{mL}$ versus $8.44 \pm 1.56 \mathrm{U} / \mathrm{mL}$ and $1.63 \pm 0.53 \mathrm{ng} / \mathrm{mL}$ versus $0.88 \pm 0.25 \mathrm{ng} / \mathrm{mL}$, respectively; both $P=0.000)$.

\section{Correlations between sRAGE and other clinical markers in critically ill patients}

Serum creatinine, IL-6, ICU duration, AGEs, and SOFA score were significantly correlated with sRAGE levels, among which AGEs levels $(r=0.558, P=0.000)$ were most strongly correlated with sRAGE levels (Table 2). Multiple linear regression analysis carried out with the above-mentioned factors set as the confounding variables indicated that only the AGEs $(P=0.000)$ and SOFA score

Table 2 Correlations between sRAGE levels and other clinical markers among critically ill patients

\begin{tabular}{lll}
\hline Variables & $\boldsymbol{R}$ & $\boldsymbol{P}$-value \\
\hline AGEs & 0.558 & 0.000 \\
Creatinine & 0.250 & 0.018 \\
HBA $_{\text {Ic }}$ & 0.074 & 0.271 \\
APACHE-II score & 0.075 & 0.267 \\
SOFA score & 0.324 & 0.003 \\
Glucose & -0.048 & 0.348 \\
C-reactive protein & 0.068 & 0.289 \\
IL-6 & 0.273 & 0.011 \\
ICU duration & 0.292 & 0.007 \\
Age & -0.007 & 0.478 \\
Sex & -0.055 & 0.325
\end{tabular}

Abbreviations: AGEs, advanced glycation end products; APACHE, acute physiologic score and chronic health evaluation; $\mathrm{HbA}_{1 \mathrm{c}}$, glycated hemoglobin; ICU, intensive care unit; IL-6, interleukin-6; SOFA, sequential organ failure assessment; sRAGE, soluble receptor for advanced glycation end products.
Table 3 Association between clinical markers and sRAGE levels among critically ill patients using multiple stepwise linear regression analysis

\begin{tabular}{llll}
\hline Variables & $\boldsymbol{\beta}$ & $\mathrm{SE}$ & $\boldsymbol{P}$-value \\
\hline AGEs & 0.558 & 0.015 & 0.000 \\
Creatinine & 0.115 & 0.001 & $0.38 \mathrm{I}$ \\
SOFA score & 0.416 & 0.033 & 0.022 \\
IL-6 & 0.105 & 0.002 & 0.357 \\
ICU duration & 0.217 & 0.009 & 0.070 \\
\hline
\end{tabular}

Note: $\beta$ indicates the standardized regression coefficient.

Abbreviations: AGEs, advanced glycation end products; ICU, intensive care unit; IL-6, interleukin-6; SOFA, sequential organ failure assessment; SE, standard error; sRAGE, soluble receptor for advanced glycation end products.

$(P=0.000)$ were significantly associated with higher sRAGE levels (Table 3 ).

\section{Association of sRAGE levels and clinical markers with prognosis}

Logistic regression analysis indicated that patient 28-day mortality rate was significantly correlated with glucose levels (odds ratio $=2.934, P=0.024$ ) and APACHE-II score (odds ratio $=1.466, P=0.038$ ). Other clinical markers, such as AGEs and sRAGE, were not shown to be influential in patient prognosis (both $P>0.05$; Table 4 ).

\section{Discussion}

Findings from this study reveal that on admission to ICU, critically ill patients with hyperglycemia display elevated circulating levels of AGEs and sRAGE. More important, the AGEs level was significantly and positively correlated with sRAGE after adjustment of other factors, suggesting the rise in plasma sRAGE levels may contribute to the accumulated AGE levels in critically ill patients. Previous clinical studies have found similar correlations between sRAGE and AGE expression levels. In patients with type 2 diabetes, serum sRAGE was correlated with serum AGEs. ${ }^{17}$ In patients without diabetes, Yamagishi et al found that sRAGE levels were associated with body mass index, alcohol intake, waist circumference, and AGEs. In addition, the association between serum sRAGE and AGEs remained statistically significant after adjustment of other factors. ${ }^{18}$

Table 4 Association of sRAGE levels and clinical markers with prognosis in critically ill patients

\begin{tabular}{llll}
\hline Variables & OR & $\mathbf{9 5 \%} \mathbf{C l}$ & P-value \\
\hline APACHE-II score & 1.466 & $1.021-2.106$ & 0.038 \\
Glucose & 2.834 & $1.148-6.997$ & 0.024 \\
sRAGE & 0.070 & $0.001-5.002$ & 0.222 \\
AGEs & 2.086 & $0.527-8.247$ & 0.295 \\
\hline
\end{tabular}

Abbreviations: AGEs, advanced glycation end products; APACHE, acute physiologic score and chronic health evaluation; $\mathrm{Cl}$, confidence interval; OR, odds ratio; sRAGE, soluble receptor for advanced glycation end products. 
Several studies investigating sRAGE levels in critically ill patients have been performed in recent years. A previous study examined serum sRAGE levels in 33 patients with community-acquired pneumonia and sepsis and performed analyses with APACHE-II and SOFA scores. Serum sRAGE levels were significantly higher among these ill patients and were a predictor of disease prognosis. ${ }^{19}$ In patients with acute lung injury, plasma sRAGE is related to the severity and poor prognosis of lung injury. ${ }^{20}$ Similarly, in patients recovering from lung transplantation, higher plasma sRAGE levels were related to prolonged ICU stay and poorer outcomes. ${ }^{21}$ Other studies have demonstrated that in critically ill patients with sepsis, sRAGE levels increased within 24 hours of admission to the ICU, and this increase was more substantial in patients who died later, suggesting a link between sRAGE levels and mortality. ${ }^{22}$ It was also observed that sRAGE levels were positively correlated with high mobility group box 1 expression, which is a RAGE ligand released from activated immune cells..$^{20}$ In addition, Arabi et al found that sRAGE levels were not only significantly increased in critically ill patients without diabetes but also correlated with serum creatinine, IL-6, and high mobility group box 1 levels, although none of these factors independently predicted the 28-day prognosis. ${ }^{23}$ Collectively, these findings are consistent with the observations in the present study. However, our results do not reflect a significant association between sRAGE levels and mortality, which might be due to differences in case distributions and mortality rates between different study centers. AGEs levels and SOFA scores were still positively associated with sRAGE, independent of other clinical factors. Taken together, these results suggest that sRAGE levels may not fully reflect the actual accumulation of AGEs. Further investigations with a larger patient population and different subtypes of critical diseases are warranted to confirm the findings and to explore the correlations between sRAGE and other biomarkers.

AGEs accumulation may be a cause and a consequence of oxidative stress and inflammation. In critically ill patients with stress hyperglycemia, high blood glucose levels can stimulate the formation and accumulation of AGEs, and in turn, induce oxidative stress and inflammatory pathways, which may further stimulate AGEs production, leading to a continuous cycle and serious pathological changes. Other clinical studies have already found increased AGEs accumulation in patients with vascular disease and diabetic complications. ${ }^{23-27}$ Epidemiologic studies have linked circulating AGEs levels to markers of inflammation, endothelial dysfunction, and diabetic vascular complications. ${ }^{10,24,25}$ Miura et al observed that endogenous secretory RAGE levels were positively correlated to specific types of AGEs in patients with type 1 diabetes. ${ }^{26}$ In addition, one study has shown that AGEs levels may predict the patient outcome of women diagnosed with type 2 diabetes, indicating that circulating AGEs may be used as a biomarker for long-term prognosis in diabetic and vascular complications. ${ }^{27}$ Greven et al observed acute accumulation of AGEs in non-diabetic critically ill patients in ICU, but AGEs levels did not correlate to APACHE-II score, ICU duration, or mortality. ${ }^{28}$ In this study, we examined the relationship between IL-6, an inflammatory marker, and sRAGE. We found a significant correlation using the Pearson's correlation analysis, but there was no significant association between the two data sets after adjustment of other clinical factors. $\mathrm{HbA}_{1 \mathrm{c}}$ is one of several early glycation products and is a biomarker of blood glucose levels. ${ }^{29}$ In our study, we did not find a significant relationship between $\mathrm{SRAGE}$ and $\mathrm{HbA}_{1 \mathrm{c}}$, which might be due to different mechanisms on how $\mathrm{HbA}_{1 \mathrm{c}}$ and AGEs are glycated and processed.

There were several limitations of this study. First, the study was conducted in a single center with relatively small sample size. Therefore, the generalizability of our findings needs further evaluation. However, this reduces possible variations between study centers, which was a common issue in multicenter studies. ${ }^{30}$ Second, critically ill patients were enrolled from a heterogeneous population. In this study, our cases covered several different and common types of critical illnesses found in the ICU. Although we excluded diabetic patients, some cardiovascular and/or renal morbidity of these patients may have still in part affected the sRAGE levels. Third, we did not investigate esRAGE, another splice variant secretory RAGE receptor, which accounts for only $20 \%$ of the sRAGE levels ${ }^{3}$ in adults. In addition, this study did not take into account the factors that could affect AGEs accumulation and sRAGE expression, such as patient conditions and diet influence. ${ }^{31}$

\section{Conclusion}

In summary, we found that serum levels of sRAGE are elevated in critically ill patients with stress hyperglycemia, and the elevated sRAGE might be associated with increased accumulation of AGEs. These observations suggest that serum sRAGE levels are not an independent predictor of 28-day mortality in critically ill patients. Further studies are needed to confirm the clinical significance of the AGEsRAGE-sRAGE axes in critically ill patients.

\section{Acknowledgment}

The authors regret to inform about the passing of Dr Cai prior to publication. The authors are grateful to Dr Cai for his expert assistance in preparation of this manuscript. 


\section{Disclosure}

The authors declare no conflicts of interest involved in this paper.

\section{References}

1. Dai L. Advanced glycation end products and diabetic vascular complications. J Int Pathol Clin Med. 2012;32:30-34.

2. Baynes JW, Thorpe SR. Glycoxidation and lipoxidation in atherogenesis. Free Radic Biol Med. 2000;28:1708-1716.

3. Wang D, Huang Q. The role of sRAGE in ligand-RAGE axis-mediated diseases. Guangdong Med J. 2011;32:1201-1204.

4. Yamagishi S, Matsui T. Soluble form of a receptor for advanced glycation end products (sRAGE) as a biomarker. Front Biosci (Elite Ed). 2010;2:1184-1195.

5. Goldin A, Beckman JA, Schmidt AM, Creager MA. Advanced glycation end products: sparking the development of diabetic vascular injury. Circulation. 2006;114:597-605.

6. Bierhaus A, Humpert PM, Morcos M, et al. Understanding RAGE, the receptor for advanced glycation end products. J Mol Med (Berl). 2005; 83:876-886.

7. Hudson BI, Carter AM, Harja E, et al. Identification, classification, and expression of RAGE gene splice variants. FASEB J. 2008;22: $1572-1580$

8. Nin JW, Ferreira I, Schalkwijk CG, et al; EURODIAB Prospective Complications Study Group. Levels of soluble receptor for AGE are cross-sectionally associated with cardiovascular disease in type 1 diabetes, and this association is partially mediated by endothelial and renal dysfunction and by low-grade inflammation: the EURODIAB Prospective Complications Study. Diabetologia. 2009;52:705-714.

9. Nin JW, Jorsal A, Ferreira I, et al. Higher plasma soluble Receptor for Advanced Glycation End Products (sRAGE) levels are associated with incident cardiovascular disease and all-cause mortality in type 1 diabetes: a 12-year follow-up study. Diabetes. 2010;59:2027-2032.

10. Goh SY, Cooper ME. Clinical review: the role of advanced glycation end products in progression and complications of diabetes. $J$ Clin Endocrinol Metab. 2008;93:1143-1152.

11. Falcone C, Emanuele E, D'Angelo A, et al. Plasma levels of soluble receptor for advanced glycation end products and coronary artery disease in nondiabetic men. Arterioscler Thromb Vasc Biol. 2005;25: 1032-1037.

12. Brownlee M. The pathobiology of diabetic complications: a unifying mechanism. Diabetes. 2005;54:1615-1625.

13. Wang J, Wang X, Di S, et al. Risk factors of stress hyperglycemia in critically ill patients and their influences on the prognosis. Clin Focus. 2012;27:238-239.

14. Schmidt AM, Yan SD, Yan SF, et al. The multiligand receptor RAGE as a progression factor amplifying immune and inflammatory responses. J Clin Invest. 2001;108:949-955.

15. Takeuchi M, Makita Z, Bucala R, Suzuki T, Koike T, Kameda Y. Immunological evidence that non-carboxymethyllysine advanced glycation end-products are produced from short chain sugars and dicarbonyl compounds in vivo. Mol Med. 2000;6:114-125.
16. Christiansen C, Toft P, Jørgensen HS, Andersen SK, Tønnesen E. Hyperglycaemia and mortality in critically ill patients. A prospective study. Intensive Care Med. 2004;30:1685-1688.

17. Nakamura K, Yamagishi S, Adachi H, et al. Serum levels of soluble form of receptor for advanced glycation end products (sRAGE) are positively associated with circulating AGEs and soluble form of VCAM-1 in patients with type 2 diabetes. Microvasc Res. 2008;76:52-56.

18. Yamagishi S, Adachi H, Nakamura K, et al. Positive association between serum levels of advanced glycation end products and the soluble form of receptor for advanced glycation end products in nondiabetic subjects. Metabolism. 2006;55:1227-1231.

19. Narvaez-Rivera RM, Rendon A, Salinas-Carmona MC, Rosas-Taraco AG. Soluble RAGE as a severity marker in community acquired pneumonia associated sepsis. BMC Infect Dis. 2012;12:15.

20. Calfee CS, Ware LB, Eisner MD, et al; NHLBI ARDS Network. Plasma receptor for advanced glycation end products and clinical outcomes in acute lung injury. Thorax. 2008;63:1083-1089.

21. Calfee CS, Budev MM, Matthay MA, et al. Plasma receptor for advanced glycation end-products predicts duration of ICU stay and mechanical ventilation in patients after lung transplantation. $J$ Heart Lung Transplant. 2007;26:675-680.

22. Bopp C, Hofer S, Weitz J, et al. sRAGE is elevated in septic patients and associated with patients outcome. J Surg Res. 2008;147:79-83.

23. Arabi YM, Dehbi M, Rishu AH, et al. sRAGE in diabetic and nondiabetic critically ill patients: effects of intensive insulin therapy. Crit Care. 2011;15:R203.

24. Accacha S, Rosenfeld W, Jacobson A, et al. Plasma advanced glycation end products (AGEs), receptors for AGEs and their correlation with inflammatory markers in middle school-age children. Horm Res Paediatr. 2013;80(5):318-327.

25. Poulsen MW, Bak MJ, Andersen JM, et al. Effect of dietary advanced glycation end products on postprandial appetite, inflammation, and endothelial activation in healthy overweight individuals. Eur J Nutr. 2014;53(2):661-672.

26. Miura J, Yamamoto Y, Osawa M, et al. Endogenous secretory receptor for advanced glycation endproducts levels are correlated with serum pentosidine and CML in patients with type 1 diabetes. Arterioscler Thromb Vasc Biol. 2007;27:253-254.

27. Kilhovd BK, Juutilainen A, Lehto S, et al. Increased serum levels of advanced glycation endproducts predict total, cardiovascular and coronary mortality in women with type 2 diabetes: a population-based 18 year follow-up study. Diabetologia. 2007;50:1409-1417.

28. Greven WL, Smit JM, Rommes JH, Spronk PE. Accumulation of advanced glycation end (AGEs) products in intensive care patients: an observational, prospective study. BMC Clin Pathol. 2010;10:4.

29. Vlassara H, Uribarri J, Cai W, et al. Effects of sevelamer on HbA1c, inflammation, and advanced glycation end products in diabetic kidney disease. Clin J Am Soc Nephrol. 2012;7(6):934-942.

30. Kavanagh BP, Mc Cowen KC. Glycemic control in the ICU. $N$ Engl $J$ Med. 2010;363(26):2540-2546.

31. Meerwaldt R, Links TP, Graaff R, et al. Increased accumulation of skin advanced glycation end-products precedes and correlates with clinical manifestation of diabetic neuropathy. Diabetologia. 2005;48:1637-1644.
Clinical Interventions in Aging

\section{Publish your work in this journal}

Clinical Interventions in Aging is an international, peer-reviewed journal focusing on evidence-based reports on the value or lack thereof of treatments intended to prevent or delay the onset of maladaptive correlates of aging in human beings. This journal is indexed on PubMed Central, MedLine,

\section{Dovepress}

CAS, Scopus and the Elsevier Bibliographic databases. The manuscript management system is completely online and includes a very quick and fair peer-review system, which is all easy to use. Visit http://www.dovepress. com/testimonials.php to read real quotes from published authors. 